\title{
Virtual coupling design for stability and transparency of multi-device haptic systems with delays *
}

\author{
Gianni Bianchini $^{1 \dagger} \quad$ Domenico Prattichizzo ${ }^{1,2} \ddagger$ \\ ${ }^{1}$ Dipartimento di Ingegneria dell'Informazione e Scienze Matematiche, Università di Siena, Italy \\ ${ }^{2}$ Advanced Robotics Department, Istituto Italiano di Tecnologia, Genova, Italy
}

\begin{abstract}
This paper deals with haptic systems involving multiple human operators and devices with computational and communication delays. A method is proposed for the design of stabilizing controllers which also guarantee transparency, i.e., controllers are designed to mitigate the impact of delays and of the controllers themselves on the realism of the tactile interaction. The proposed approach exploits an extension of a previously developed passivity-based framework for stabilization combined with a special loop shaping-like technique. The design procedure involves the solution of a sequence of Linear Matrix Inequality (LMI) optimization problems.
\end{abstract}

\section{INTRODUCTION}

One of the main issues in stability analysis for haptics is that the human operator handling the haptic interface is difficult to model. A well-established approach is to consider the human operator as an unmodeled passive system and to design controllers to guarantee passivity of suitable components of the haptic loop, as shown in the seminal paper [9] and more recently in [21], where the authors provide different approaches to the characterization of passivity in sampled-data systems and in particular in haptics. The first results on stability of the haptic loop were formalized for a single-degreeof-freedom (DoF) contact interaction, which has the advantage of providing very elegant closed form conditions [10]. On the other hand, recent applications of haptics involve multi-point contact interaction with the environment using several devices, and hence the single-DoF approach turns out to be inadequate [2]. This paper deals with multi-DoF haptic systems where one or more human operators interact with a shared virtual environment through multiple devices.

Several approaches have been proposed to address stability of multi-DoF force feedback loops [12],[13]. These approaches share the common idea of monitoring the energy flowing into the system and dissipating part of it with different strategies to guarantee passivity constraints. A similar method has been presented in [19], where the control is based on an energy observer.

Our approach is different in nature since it does not need to monitor the energy and tune the dissipating action of the controller accordingly. On the contrary, our method is more related to $[14,16]$, where structural conditions are given for the controller (referred to as virtual coupling) to guarantee the stability of the haptic loop for the single-DoF case, and to [17], in which an $H_{\infty}$ approach to transparent controller design is proposed for the case of a single one-DOF operator. This paper builds upon previous contributions $[5,6,7]$, in which a framework is proposed based on passivity and Linear Matrix Inequalities (LMIs) for stabilizing controller design in haptic loops involving multiple devices and human operators. In particular, we extend these results taking into account the presence

${ }^{*}$ This work was partially supported by the European Commission with the Collaborative Project no. 288917 "Devices for Assisted Living" (DALi), within the FP7-ICT-2011-7-5.4 program "Cognitive Systems and Robotics".

†e-mail: giannibi@dii.unisi.it

†e-mail: domenico.prattichizzo@unisi.it

IEEE World Haptics Conference 2013

14-18 April, Daejeon, Korea

978-1-4799-0088-6/13/\$31.00 @2013 IEEE of constant time delays, which may arise both in the computations for the simulation of the virtual environment [3] and in communication, due to the fact that the virtual environment may be shared among several remote users [20]. Moreover, we address the performance of controllers in terms of transparency. When delays are present, the controller has to meet the requirement of limiting the impact of both the controller itself and the delays on the realism of interaction. The transparency property is formulated in an $H_{\infty}$ context as a model matching problem, extending the formulation of controller transparency given in [17] and [7] for the undelayed case. The model matching problem arising in the multi-device delayed scenario considered here is solved using a loop shaping-like technique. The resulting design procedure involves the solution of a sequence of LMI optimizations.

The paper is organized as follows. In Section 2 we report some preliminary results; in Section 3 we formalize the problems of stability and transparency; in Section 4 we address controller parameterization while in Section 5 we solve the problem of controller design for transparency. Section 6 presents the experimental validation of the proposed results and conclusions are finally drawn in Section 7.

\section{Notation}

For a square matrix $X, X>0(X<0)$ denotes positive (negative) definiteness, $X^{T}$ denotes transpose and $\|X\|$ some matrix norm. $I\left(I_{m}\right)$ is the $(m \times m)$ identity matrix. $X=$ blockdiag $\left(X_{1}, \ldots, X_{N}\right)$ denotes a block-diagonal matrix with diagonal blocks $X_{1}, \ldots, X_{N}$. With $\mathscr{B} \mathscr{D}\left(m ; m_{1}, \ldots, m_{N}\right)$ we denote the set of $m \times m$ block-diagonal matrices whose $N$ blocks have dimensions $m_{1} \times m_{1}, \ldots, m_{N} \times m_{N}$, with $\sum_{i=1}^{N} m_{i}=m$. The latter notation is also used without ambiguity for block-diagonal transfer matrices of $m$-input, $m$-output linear systems and, more generally, of $m$-input, $m$-output operators. The notation $G:\left(A_{G}, B_{G}, C_{G}, D_{G}\right)$ indicates that the linear time invariant system $G$ has a state-space representation $\left(A_{G}, B_{G}, C_{G}, D_{G}\right)$. For a complex matrix $X, \bar{\sigma}[X]$ and $\sigma[X]$ denote its maximum and minimum singular values, while for the transfer matrix $G(z)$ of a linear discrete-time system operating on signals sampled with period $T,\|G\|_{\infty}$ denotes its $H_{\infty}$ norm, i.e., $\|G\|_{\infty}=\sup _{\omega} \bar{\sigma}\left[G\left(e^{j \omega T}\right)\right]$. For brevity, we sometimes denote $G(z)$ with $G$ and $\bar{\sigma}\left[G\left(e^{j \omega T}\right)\right]$ simply with $\bar{\sigma}[G]$ without ambiguity.

\section{Preliminaries}

The results in this paper exploit a slight generalization [6] of standard passivity concepts [8].

Definition 1 (continuous-time passivity). Let $\Sigma$ be a continuoustime dynamical system with input $u(t) \in \mathbb{R}^{m}$, output $y(t) \in \mathbb{R}^{m}$, and state $\psi(t) \in \mathbb{R}^{n}$. If there exists a continuously differentiable positive definite function $\mathscr{V}(\psi): \mathbb{R}^{n} \rightarrow \mathbb{R}$ (called the storage function) and $m \times m$ symmetric matrices $\Delta$ and $\Phi$ such that along all system trajectories $(\psi(t), u(t), y(t)), t \in \mathbb{R}$, the following inequality holds

$$
\dot{\mathscr{V}}(\psi(t))<y(t)^{T} u(t)-y(t)^{T} \Delta y(t)-u(t)^{T} \Phi u(t),
$$

then, system $\Sigma$ is passive if $\Delta=\Phi=0$, output strictly passive with level $\Delta(\Delta-$ OSP) if $\Delta>0, \Phi=0$, input strictly passive with level $\Phi(\Phi-$ ISP) if $\Delta=0, \Phi>0$, respectively. 
Definition 2 (discrete-time passivity). Let $\Sigma_{d}$ be a discrete-time dynamical system with input $u(k) \in \mathbb{R}^{m}$, output $y(k) \in \mathbb{R}^{m}$, and vector $\psi(k) \in \mathbb{R}^{n}$. If there exists a positive definite function $\mathscr{V}(\psi)$ : $\mathbb{R}^{n} \rightarrow \mathbb{R}$ and $m \times m$ symmetric matrices $\Delta$ and $\Phi$ such that along all system trajectories $(\psi(k), u(k), y(k)), k \in \mathbb{N}$, this inequality holds

$\mathscr{V}(\psi(k+1))-\mathscr{V}(\psi(k))<y(k)^{T} u(k)-y(k)^{T} \Delta y(k)-u(k)^{T} \Phi u(k)$,

then the system is passive if $\Delta=\Phi=0$, output strictly passive $(\Delta-$ OSP $)$ if $\Delta>0, \Phi=0$, input strictly passive $(\Phi-$ ISP $)$ if $\Delta=$ $0, \Phi>0$, respectively.

Note that $\Delta$ and $\Phi$ need not necessarily be positive definite: indeed, $\Sigma, \Sigma_{d}$ are said to lack OSP (ISP) when the above definitions hold for non-positive definite $\Delta(\Phi)$.

Let $\Sigma_{d}:(A, B, C, D)$, where $A \in \mathbb{R}^{n \times n}, B \in \mathbb{R}^{n \times m}, C \in \mathbb{R}^{m \times n}$, $D \in \mathbb{R}^{m \times m}$. A straightforward extension of the Kalman-YacubovićPopov lemma [8] holds.

Lemma 1 The discrete-time system $\Sigma_{d}$ is passive ( $\Delta-O S P$, $\Phi-I S P)$ if and only if there exists a symmetric matrix $P \in \mathbb{R}^{n \times n}$ such that the following matrix inequalities hold:

$$
\begin{aligned}
& P>0 \\
& {\left[\begin{array}{ll}
A^{T} P A-P+C^{T} \Delta C & A^{T} P B-\frac{C^{T}}{2}+C^{T} \Delta D \\
B^{T} P A-\frac{C}{2}+D^{T} \Delta C & B^{T} P B-\frac{D+D^{T}}{2}+D^{T} \Delta D+\Phi
\end{array}\right]<0 .}
\end{aligned}
$$

It is convenient to formulate the passivity condition of Lemma 1 in terms of LMIs plus an inversion constraint. We have the following result (see [5]).

Lemma 2 Let $\Delta>0 . \Sigma_{d}$ is passive $(\Delta-O S P(\Delta>0), \Phi-I S P)$ if and only if there exists a symmetric matrix $Q \in \mathbb{R}^{n \times n}$ satisfying

$$
\begin{aligned}
& Q>0, \quad\left[\begin{array}{cc}
Y & R^{T} \\
R & S
\end{array}\right]>0, \\
& R=\left[\begin{array}{c}
\frac{C}{2} \\
A \\
C
\end{array}\right], \quad S=\left[\begin{array}{ccc}
\frac{D^{T}+D}{2}-\Phi & B^{T} & D^{T} \\
B & Q & 0 \\
D & 0 & \Sigma
\end{array}\right], \\
& Y=Q^{-1}, \quad \Sigma=\Delta^{-1} .
\end{aligned}
$$

Finally, we need the following characterization of the $H_{\infty}$ norm of a linear discrete-time system [18], which is also an LMI plus an inversion constraint.

Lemma 3 Consider a linear time invariant discrete-time system $G:(A, B, C, D)$, and let $\mu$ be a positive scalar. Then, $\|G\|_{\infty}<\mu$ if and only if there exist symmetric matrices $X$ and $U$ such that

$$
\begin{aligned}
& {\left[\begin{array}{cccc}
X & 0 & A^{T} & C^{T} \\
0 & \mu^{2} I & B^{T} & D^{T} \\
A & B & U & 0 \\
C & D & 0 & I
\end{array}\right]>0} \\
& X=U^{-1}
\end{aligned}
$$

\section{Problem formulation}

A haptic system is typically modeled as a sampled-data interconnected system (with sampling period $T$ ) as in Fig. 1. The main components are a human operator block $H$, a haptic device block $D$, a computer-simulated virtual environment $E$, and a virtual coupling $V$, whose role is to act as a controller [9], [14]-[16]. The mappings $H$ and $D$ are continuous-time, while $E$ and $V$ are discretetime. In this paper, we consider $N$ human operators $H_{i}, i=1, \ldots, N$, where each $H_{i}$ is assumed to have $m_{i}$ DoF. Each human operator $H_{i}$ is assumed to interact with a set of $M_{i}$ devices, denoted $d_{i, j}$, $j=1, \ldots, M_{i}$ and having $m_{i, j}$ DoF, with $m_{i}=\sum_{j=1}^{M_{i}} m_{i, j}$. The interaction of each human operator $H_{i}$ with the respective set of devices

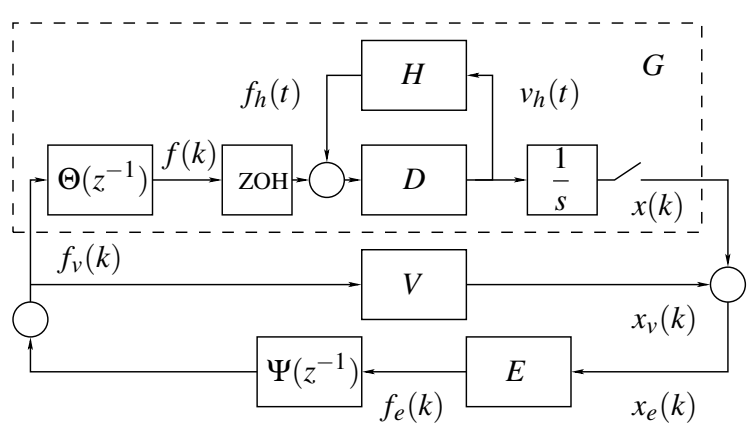

Figure 1: Haptic loop

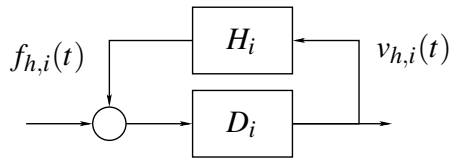

Figure 2: Human-device interconnection

is therefore modeled by the feedback loop in Fig. 2, where

$$
D_{i}=\operatorname{blockdiag}\left(d_{i, 1}, \ldots d_{i, M_{i}}\right)
$$

and where $f_{h, i}(t) \in \mathbb{R}^{m_{i}}$ represents the generalized force vector and $v_{h, i}(t) \in \mathbb{R}^{m_{i}}$ is the corresponding generalized velocity vector.

Let $m=\sum_{i=1}^{N} m_{i}$. It turns out that the overall human-device interconnection is described by the $H-D$ loop in Fig. 1, where

$$
\begin{gathered}
H=\operatorname{blockdiag}\left(H_{1}, \ldots, H_{N}\right) \in \mathscr{B} \mathscr{D}\left(m ; m_{1}, \ldots, m_{N}\right), \\
D=\operatorname{blockdiag}\left(D_{1}, \ldots, D_{N}\right) \in \mathscr{B} \mathscr{D}\left(m ; m_{1}, \ldots, m_{N}\right), \\
f_{h}(t)=\left[f_{h, 1}^{T}(t) \ldots f_{h, N}^{T}(t)\right]^{T}, \\
v_{h}(t)=\left[v_{h, 1}^{T}(t) \ldots v_{h, N}^{T}(t)\right]^{T} .
\end{gathered}
$$

Remark 1 The structure of the blocks $H$ and $D$ reflects different ways in which the human operators interact with the set of devices. For instance, two devices may be operated by one (e.g., with both hands) or two humans. Each particular structure can be exploited as in [6] in order to reduce the conservatism of the approach proposed here, but this fact is not taken into account in order to avoid excessive technicalities.

Let $x(k) \in \mathbb{R}^{m}$ and $f(k) \in \mathbb{R}^{m}$ denote the sampled generalized device displacement vector and the sampled generalized force feedback vector, respectively.

We account for the presence of constant delays in the haptic loop via the blocks $\Theta\left(z^{-1}\right)$ and $\Psi\left(z^{-1}\right)$ in Fig. 1. In their most common interpretation, $\Psi\left(z^{-1}\right)$ models computational delays in the simulation of the virtual environment while $\Theta\left(z^{-1}\right)$ accounts for delays in the transmission of information between the simulator and the devices. This is the standard paradigm used, e.g., in [15],[16]. We assume such blocks to have the form

$$
\begin{aligned}
& \Theta\left(z^{-1}\right)=\operatorname{blockdiag}\left(z^{-\theta_{1}} I_{m_{1}}, \ldots, z^{-\theta_{N}} I_{m_{N}}\right) \\
& \Psi\left(z^{-1}\right)=\operatorname{blockdiag}\left(z^{-\psi_{1}} I_{m_{1}}, \ldots, z^{-\psi_{N}} I_{m_{N}}\right)
\end{aligned}
$$

with $\theta_{i}, \psi_{i} \in \mathbb{N}, i=1, \ldots, N$.

Remark 2 The structure (4),(5) of $\Theta\left(z^{-1}\right)$ and $\Psi\left(z^{-1}\right)$ implies that all devices in the device block $D_{i}$ pertaining to the human operator $H_{i}$ are affected by the same delay, which is likely to happen in most cases. Although different delays for all devices can be accounted for, we prefer not to consider this case to keep the notation simple.

Remark 3 A more accurate model of the delays would in principle require the presence of a transmission delay block affecting 
the measurement of the device displacements made available to the virtual environment simulator. Accounting for this fact involves additional technicalities and will be addressed in a future work.

Let $G$ be the portion of the loop encircled by the dashed line in Fig. 1 and let $L$ denote the overall haptic loop. In this paper, we address the problem of designing the virtual coupling $V$ in order to achieve stability and transparency, as explained below.

\subsection{Stability}

The stability requirement is fundamental to prevent oscillatory behaviour of the haptic system during interaction. In this respect, we consider the notion of haptic loop stability introduced in [15].

Definition 3 The haptic loop $L$ is stable if the generalized velocity vector $v_{h}(t)$ goes to zero in steady state.

\subsection{Transparency}

An important performance index in a haptic system is the transparency of components such as the virtual coupling and the delays with respect to the virtual environment. When delays are present and/or a virtual coupling is needed to guarantee stability, it is desirable that their effect on the dynamics of the virtual environment, as it is perceived by the human, be mitigated. This amounts to the requirement that the admittance of the loop between the sampled displacement $x$ and the force feedback $f$ (Fig. 1) be as close as possible to ("match") the admittance of the ideal virtual environment according to some criterion, under the stability constraint. We find it convenient to formulate the transparency requirement as an $H_{\infty}$ model matching problem.

The admittance between $x$ and $f$ is given by

$$
\mathscr{F}=\Theta(I+\Psi E V)^{-1} \Psi E .
$$

To be as general as possible, we consider good transparency to be achieved when the frequency response (in both amplitude and phase) of $\mathscr{F}$ closely matches that of a delayed version of the virtual environment $E$ in a prescribed frequency range (typically at low frequencies, depending on the required bandwidth). Matching $\mathscr{F}$ with a delayed version of $E$, where the amount of delay is given as a design parameter, introduces flexibility in trading off between the compensation of phase delay through a derivative action and keeping the strength of the control introduced by the virtual coupling low. Indeed, a strong control action typically has undesirable effects such as reducing the perceived stiffness of the environment. Motivated by the observations above, we consider the delay block

$$
\Lambda(z)=\operatorname{blockdiag}\left(z^{-\lambda_{1}} I_{m_{1}}, \ldots, z^{-\lambda_{N}} I_{m_{N}}\right),
$$

where $\lambda_{i} \in \mathbb{N}, 0 \leq \lambda_{i} \leq \theta_{i}+\psi_{i} \forall i=1, \ldots, N$ are given as design parameters, and introduce the "mismatch" transfer function:

$$
\mathscr{M}=\mathscr{F}-\Lambda E=\Theta(I+\Psi E V)^{-1} \Psi E-\Lambda E .
$$

The transparency requirement then boils down to making $\mathscr{M}$ "small" in a given frequency range, i.e., enforcing

$$
\bar{\sigma}[\mathscr{M}]<\mu \quad \forall \omega \leq \omega_{0}
$$

for given $\omega_{0}$ and given (possibly minimum) $\mu$, subject to closedloop stability. tion.

The problem of ensuring stability is addressed in the next sec-

\section{STABILIZING VIRTUAL COUPLING PARAMETERIZATION}

In this section we provide the parameterization of a set $\mathbf{S}$ of stabilizing controllers (virtual couplings) which turns out to be computationally effective for addressing the transparency optimization problem. The results that follow extend the passivity-based framework proposed in [6] to account for the presence of delays, but neglecting the relaxations used to address the possible different structures of the $H-D$ block (see Remark 1).



Figure 3: Transformed haptic loop $\hat{L}$

As in most approaches to stability analysis of haptic systems, we rely on the assumption that both the human and the device enjoy the passivity property; in particular, the human operator is passive but otherwise unmodeled, while the device dynamics is assumed to be OSP [15]. The OSP level of a given device is related to the damping introduced into the system by the device itself. The problem of its computation has been dealt with in [14] for linear and in [16] for nonlinear devices. In this respect, we remark that the approach in this paper does not require an explicit (linear or nonlinear) model of the devices, since only their passivity levels are involved. We therefore make the following assumption.

Assumption 1 (a) Each device $d_{i, j}$ is a $\Delta_{d_{i, j}}$-OSP (but otherwise unmodeled) continuous-time system, and (b) each human operator block $H_{i}$ is a passive (unmodeled) continuous-time $m_{i}$-input, $m_{i^{-}}$ output operator.

In view of Assumption 1, it is easily seen [5] that the device block $D$ defined as in (3) is $\Delta_{D}$-OSP, where

$$
\begin{aligned}
& \Delta_{D}=\operatorname{blockdiag}\left(\Delta_{D_{1}}, \ldots, \Delta_{D_{N}}\right), \\
& \Delta_{D_{i}}=\operatorname{blockdiag}\left(\Delta_{d_{i, 1}}, \ldots, \Delta_{d_{i, M_{i}}}\right) .
\end{aligned}
$$

We now look for a stability criterion in the sense of Definition 3. To this purpose, we exploit a generalization of the approach in [14],[16]. To proceed, consider the transformed loop $\hat{L}$ depicted in Fig. 3, where

$$
\begin{aligned}
& \hat{G}=\frac{z-1}{T z}\left[G+\hat{\Theta}\left(z^{-1}\right)\right], \hat{V}=\frac{z-1}{T z}\left[V-\hat{\Theta}\left(z^{-1}\right)\right], \\
& \hat{E}=\frac{T z}{z-1} \Psi\left(z^{-1}\right) E
\end{aligned}
$$

where $\hat{\Theta}\left(z^{-1}\right)$ is the filter

$$
\hat{\Theta}\left(z^{-1}\right)=\frac{T}{2} \text { blockdiag }\left(\vartheta_{1}\left(z^{-1}\right) \Delta_{D_{1}}^{-1}, \ldots, \vartheta_{N}\left(z^{-1}\right) \Delta_{D_{N}}^{-1}\right),
$$

being $\vartheta_{i}\left(z^{-1}\right)=1+z^{-1}+\cdots+z^{-\theta_{i}}, \quad i=1, \ldots, N$.

The loop $\hat{L}$ is a discrete-time system whose dynamics are the same as those of the original loop $L$. In the transformed loop, the blocks $\hat{G}, \hat{V}$ and $\hat{E}$ can be characterized in terms of their OSP or ISP levels. The following result states the passivity properties of $\hat{G}$. The proof follows from a quite straightforward extension of Lemma 2 in [16] and is omitted for brevity.

Theorem 1 Let $\hat{G}$ be as in (11) and suppose Assumption 1 holds. Assume $\Delta_{D}$ as in (10). Then $\hat{G}$ is (discrete-time) $\Delta_{\hat{G}}-O S P$ with

$$
\Delta_{\hat{G}}=\Delta_{D} .
$$

The loop transformation introduced above is a simple generalization of the one employed in [15] and by an argument similar to the one used there, the following stability condition is readily obtained.

Theorem 2 If the transformed loop $\hat{L}$ is asymptotically stable, then the haptic loop L is stable in the sense of Definition 3, i.e., the generalized velocity vector $v_{h}(t)$ goes to zero in steady state. 
The next step is to assess stability of the transformed loop $\hat{L}$ by means of linear matrix inequalities involving the passivity levels of $\hat{G}, \hat{V}$ and $\hat{E}$. The following condition generalizes a standard result concerning stability of feedback and parallel interconnections of passive systems (see [6]).

Lemma 4 If there exist symmetric matrices $\Delta_{\hat{V}}$, $\Phi_{\hat{E}}$ such that

1. $\hat{V}$ is $\Delta_{\hat{V}}-O S P$,

2. $\hat{E}+\Phi_{\hat{E}}$ is passive, i.e., $\hat{E}$ is $\left(-\Phi_{\hat{E}}\right)-I S P$, and

3. the following matrix inequalities hold:

$$
\Delta_{\hat{V}}>0, \quad\left[\begin{array}{ll}
\Delta_{D}-\Phi_{\hat{E}} & -\Phi_{\hat{E}} \\
-\Phi_{\hat{E}} & \Delta_{\hat{V}}-\Phi_{\hat{E}}
\end{array}\right]>0
$$

then, the haptic loop L is stable.

Let $E$ be given as a discrete-time transfer function $E(z)$, and let $\left(A_{\hat{E}}, B_{\hat{E}}, C_{\hat{E}}, D_{\hat{E}}\right)$ be a state-space realization of

$$
\hat{E}=\hat{E}(z)=\frac{T z}{z-1} \Psi\left(z^{-1}\right) E(z) .
$$

The following result shows that checking the existence of $\Delta_{\hat{V}}$ and $\Phi_{\hat{E}}$ satisfying the stability condition of Lemma 4 can be cast as an LMI problem.

Theorem 3 If there exist symmetric matrices $P, \Delta_{\hat{V}}, \Phi_{\hat{E}}$ such that

1. $\hat{V}$ is $\Delta_{\hat{V}}-O S P$,

2. the following LMIs hold

$$
\begin{aligned}
& \Delta_{\hat{V}}>0, \\
& P>0 \\
& {\left[\begin{array}{cc}
A_{\hat{E}}^{T} P A_{\hat{E}}-P & A_{\hat{E}}^{T} P B_{\hat{E}}-\frac{C_{\hat{E}}^{T}}{2} \\
B_{\hat{E}}^{T} P A_{\hat{E}}-\frac{C_{\hat{E}}}{2} & B_{\hat{E}}^{T} P B_{\hat{E}}-\frac{D_{\hat{E}}}{2}-\frac{D_{\hat{E}}^{T}}{2}-\Phi_{\hat{E}}
\end{array}\right]<0,} \\
& {\left[\begin{array}{ll}
\Delta_{D}-\Phi_{\hat{E}} & -\Phi_{\hat{E}} \\
-\Phi_{\hat{E}} & \Delta_{\hat{V}}-\Phi_{\hat{E}}
\end{array}\right]>0,}
\end{aligned}
$$

then the haptic loop L is stable.

Proof. It follows immediately from Lemma 4 by observing that condition 2) in that Lemma is equivalent to the second and third of (15) by Lemma 1 when $E$ (and thus $\hat{E}$ ) is a linear system.

We are now ready to derive an LMI parameterization of a set $\mathbf{S}$ of stabilizing virtual coupling systems of given fixed order $\bar{n}$. Within this set, the transparency problem will be considered.

Let $\left(A_{\hat{\Theta}}, B_{\hat{\Theta}}, C_{\hat{\Theta}}, D_{\hat{\Theta}}\right)$ be a state-space representation of the filter $\hat{\Theta}\left(z^{-1}\right)$ and let $V:\left(A_{V}, B_{V}, C_{V}, D_{V}\right)$, where, $A_{V} \in \mathbb{R}^{\bar{n} \times \bar{n}}, B_{V} \in$ $\mathbb{R}^{\bar{n} \times m}, C_{V} \in \mathbb{R}^{m \times \bar{n}}, D_{V} \in \mathbb{R}^{m \times m}$. Then, $\hat{V}:\left(A_{\hat{V}}, B_{\hat{V}}, C_{\hat{V}}, D_{\hat{V}}\right)$ where

$$
\begin{gathered}
A_{\hat{V}}=\left[\begin{array}{ccc}
A_{V} & 0 & -\frac{1}{T} B_{V} \\
0 & A_{\hat{\Theta}} & -\frac{1}{T} B_{\hat{\Theta}} \\
0 & 0 & 0
\end{array}\right], \quad B_{\hat{V}}=\left[\begin{array}{c}
\frac{1}{T} B_{V} \\
\frac{1}{T} B_{\hat{\Theta}} \\
I
\end{array}\right], \\
C_{\hat{V}}=\left[\begin{array}{lll}
C_{V} & -C_{\hat{\Theta}} & -\frac{1}{T}\left(D_{V}-D_{\hat{\Theta}}\right)
\end{array}\right], \quad D_{\hat{V}}=\frac{1}{T}\left(D_{V}-D_{\hat{\Theta}}\right) .
\end{gathered}
$$

The following result yields the sought parameterization of $\mathbf{S}$.

Theorem 4 Consider the haptic loop L, let $\Delta_{D}$ be the OSP level of the devices as in (10), and let $\hat{E}:\left(A_{\hat{E}}, B_{\hat{E}}, C_{\hat{E}}, D_{\hat{E}}\right)$. Then, all controllers $V:\left(A_{V}, B_{V}, C_{V}, D_{V}\right)$ such that there exist matrices $P, \Delta_{\hat{V}}, \Phi_{\hat{E}}, \Sigma_{\hat{V}}, Q, Y$ satisfying the LMIs

$$
\begin{aligned}
& P>0, \Delta_{\hat{V}}>0, \\
& {\left[\begin{array}{cc}
A_{\hat{E}}^{T} P A_{\hat{E}}-P & A_{\hat{E}}^{T} P B_{\hat{E}}-\frac{C_{\hat{E}}^{T}}{2} \\
B_{\hat{E}}^{T} P A_{\hat{E}}-\frac{C_{\hat{E}}}{2} & B_{\hat{E}}^{T} P B_{\hat{E}}-\frac{D_{\hat{E}}}{2}-\frac{D_{\hat{E}}^{T}}{2}-\Phi_{\hat{E}}
\end{array}\right]<0,} \\
& {\left[\begin{array}{cc}
\Delta_{D}-\Phi_{\hat{E}} & -\Phi_{\hat{E}} \\
-\Phi_{\hat{E}} & \Delta_{\hat{V}}-\Phi_{\hat{E}}
\end{array}\right]>0,} \\
& Q P>0,\left[\begin{array}{cc}
Y & R^{T} \\
R & S
\end{array}\right]>0, \\
& R=\left[\begin{array}{c}
\frac{C_{\hat{V}}}{2} \\
A_{\hat{V}} \\
C_{\hat{V}}
\end{array}\right], S=\left[\begin{array}{ccc}
\frac{D_{\hat{V}}+D_{\hat{V}}^{T}}{2} & B_{\hat{V}}^{T} & D_{\hat{V}}^{T} \\
B_{\hat{V}} & Q & 0 \\
D_{\hat{V}} & 0 & \Sigma_{\hat{V}}
\end{array}\right],
\end{aligned}
$$

plus the inversion constraint

$$
\Sigma_{\hat{V}}=\Delta_{\hat{V}}^{-1}, Y=Q^{-1}
$$

stabilize the haptic loop $L$.

Proof. (17) and (18),(19),(20) ensure, respectively, that the OSP level $\Delta_{\hat{V}}$ satisfies the stability condition of Theorem 4 for some $\Phi_{\hat{E}}$ and that $V$ is $\Delta_{\hat{V}}$-OSP; the latter property follows from Lemma 2 .

\section{DESIGN FOR TRANSPARENCY}

We now consider the problem of achieving transparency as defined in Section 3.2 within the stabilizing controller class $\mathbf{S}$. A possible optimal approach is to solve the $H_{\infty}$ norm minimization problem

$$
\min _{V \in \mathbf{S}}\left\|W_{1} \mathscr{M} W_{2}\right\|_{\infty}
$$

where $W_{1}$ and $W_{2}$ are suitable weights (low-pass filters) that penalize the objective function at the frequencies of interest.

Unfortunately, solving (21) directly is not easily accomplished using convex optimization techniques, in particular when algebraic loops are present in the feedback interconnection of $\Psi E$ and $V$. In this respect, we observe that $E$ is likely to have feed-through, i.e., $D_{E} \neq 0$ (for instance, when it is obtained by backward Euler or Tustin approximation of a continuous time system), $V$ is required to have $D_{V}>0$ in order for $\hat{V}$ to be passive, and $\Psi$ may have some non-delayed components, i.e, $\psi_{i}=0$ for some $i$.

Such an approach is used in [17] but, due to the above mentioned computational difficulties, the results are limited to the case of only one single-DoF device (SISO setting). Moreover, in that paper delays are not accounted for.

Convexification techniques for $H_{\infty}$ control problems, such as those in [18], could in principle be employed in combination with the controller parameterization proposed here in order to solve (21) optimally, provided that possible algebraic loops are avoided, e.g., by introducing artificial delay in $\Psi$. Nevertheless, we prefer to pursue a simpler approach that can be used in the general case and that can be easily formulated in terms of LMI problems, at the price of obtaining sub-optimal solutions. More specifically, we will proceed as in standard MIMO loop shaping techniques by enforcing bounds on the singular values of a suitable "open-loop" response through the design of $V$ in order to achieve "closed-loop" performance, i.e., to make $\bar{\sigma}[\mathscr{M}]$ small at given frequencies. Contrary to classic loop shaping, no stability requirement has to be considered, since $V$ will be restricted to belong to the set of stabilizing controllers $\mathbf{S}$.

To proceed, let us introduce the transfer function

$$
\mathscr{L}=I+\Psi E V-\Psi \Lambda^{-1} \Theta .
$$

Note that the transfer function $\mathscr{L}(z)$ is proper under the assumptions made on $\Lambda(z)$ (see (7)). Moreover, we observe that if $\bar{\sigma}[\mathscr{L}]$ is 
small, then also $\bar{\sigma}[\mathscr{M}]$ is small, i.e.,

$$
\bar{\sigma}[\mathscr{L}] \ll 1 \Rightarrow \bar{\sigma}[\mathscr{M}] \ll 1 .
$$

Indeed, by using the matrix inversion lemma, $\mathscr{M}$ can be rewritten as

$$
\mathscr{M}=-\Lambda\left(I+\Lambda^{-1} \Theta \mathscr{L}^{-1} \Psi\right)^{-1} E .
$$

Hence, if $\bar{\sigma}[\mathscr{L}] \ll 1$ we have

$$
\begin{aligned}
\bar{\sigma}[\mathscr{M}] & \approx \bar{\sigma}\left[-\Lambda\left(\Lambda^{-1} \Theta \mathscr{L}^{-1} \Psi\right)^{-1} E\right] \\
& =\bar{\sigma}\left[-\Lambda \Psi^{-1} \mathscr{L} \Theta^{-1} \Lambda E\right]
\end{aligned}
$$

and therefore, if $\bar{\sigma}[\mathscr{L}] \ll 1$ then also $\bar{\sigma}[\mathscr{M}] \ll 1$.

Remark 4 More accurate bounds on $\bar{\sigma}[\mathscr{M}]$ with respect to $\bar{\sigma}[\mathscr{L}]$ could possibly be derived by exploiting results such as those in [4], but we believe this issue to be out of the scope of this paper.

Given the above arguments, making $\bar{\sigma}[\mathscr{M}]$ small at given frequencies can be achieved in a sub-optimal fashion by solving the optimization problem

$$
\min _{V \in \mathbf{S}}\|\tilde{\mathscr{L}}\|_{\infty}
$$

where $\tilde{\mathscr{L}}=W_{1} \mathscr{L} W_{2}$ and $W_{1}, W_{2}$ are suitable weights.

To this purpose, we observe that $\tilde{\mathscr{L}}$ has the form

$$
\tilde{\mathscr{L}}=W_{3} V W_{2}+W_{4}
$$

where $W_{3}=W_{1} \Psi E$ and $W_{4}=W_{1}\left(I-\Psi \Lambda^{-1} \Theta\right) W_{2}$. Hence, a state space representation $\left(A_{\tilde{\mathscr{L}}}, B_{\tilde{\mathscr{L}}}, C_{\tilde{L}}, D_{\tilde{L}}\right)$ of $\tilde{\mathscr{L}}$ can be parameterized affinely in the matrices $\left(A_{V}, B_{V}, C_{V}, D_{V}\right)$ in the same fashion as the parameterization of $\hat{V}$ in (16). The explicit expressions of $A_{\tilde{\mathscr{L}}}, B_{\tilde{\mathscr{L}}}, C_{\tilde{\mathscr{L}}}, D_{\tilde{\mathscr{S}}}$ are easy to compute and are not reported here due to space limitations.

Given a positive scalar $\mu$, computing a stabilizing controller $V \in \mathbf{S}$ ensuring that $\|\tilde{\mathscr{L}}\|_{\infty}<\mu$ can be accomplished by combining the parameterization of $\mathbf{S}$ in Theorem 4 and the characterization of the $H_{\infty}$ norm bound given in Lemma 3 . This boils down to solving the problem (17)-(20) with the additional variables $X$ and $U$ and the additional constraints

$$
\begin{aligned}
& \text { (a) }\left[\begin{array}{cccc}
X & 0 & A_{\tilde{\mathscr{L}}}^{T} & C_{\tilde{\mathscr{L}}}^{T} \\
0 & \mu^{2} I & B_{\tilde{\mathscr{L}}}^{T} & D_{\tilde{\mathscr{L}}}^{T} \\
A_{\tilde{\mathscr{L}}} & B_{\tilde{\mathscr{L}}} & U & 0 \\
C_{\tilde{\mathscr{L}}} & D_{\tilde{\mathscr{L}}} & 0 & I
\end{array}\right]>0, \\
& \text { (b) } X=U^{-1},
\end{aligned}
$$

where the first of (24) is an LMI in $A_{V}, B_{V}, C_{V}, D_{V}$.

Remark 5 Finding $V \in \mathbf{S}$ that ensures $\|\tilde{\mathscr{L}}\|_{\infty}<\mu$ reduces to solving the LMIs (17)-(19),(24(a)) with the additional inversion constraints (20),(24(b)). The solution can be carried out as a sequence of LMI optimization problems using the cone complementarity linearization algorithm [11]. Moreover, since the condition $\|\tilde{\mathscr{L}}\|_{\infty}<\mu$ is convex in $\mu,\|\tilde{\mathscr{L}}\|_{\infty}$ can be minimized by proceeding by bisection on $\mu$. We omit the outline of the algorithm here due to space constraints.

Remark 6 A measure of the degree of transparency achieved by the designed controller, which may be conservative, can be computed as the ratio

$$
\tau=\frac{\sup _{\omega<\omega_{0}} \bar{\sigma}\left[\mathscr{M}\left(e^{j \omega T}\right)\right]}{\inf _{\omega<\omega_{0}} \underline{\sigma}\left[E\left(e^{j \omega T}\right)\right]}
$$

where $\mathscr{M}$ is the mismatch function corresponding to the computed controller. This index (the lower, the better) gives an idea of the

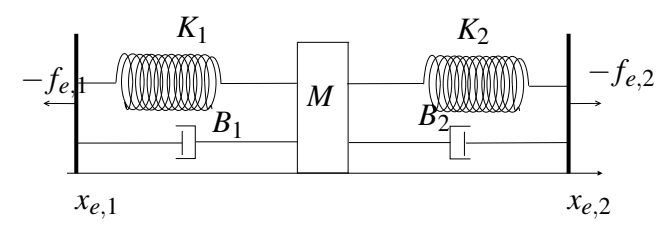

Figure 4: Virtual environment model

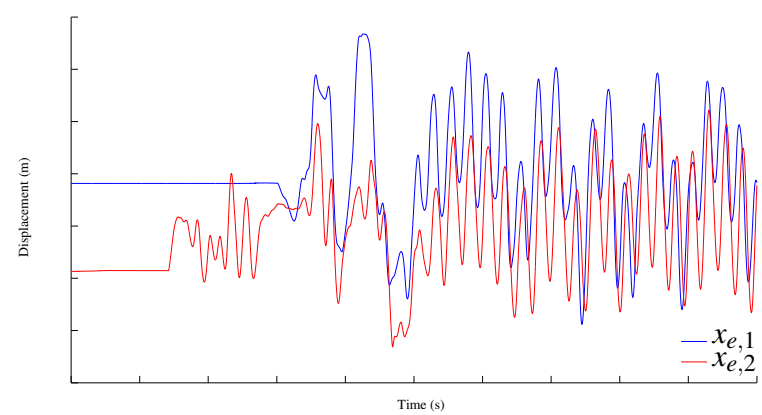

Figure 5: Device displacements during a run without virtual coupling

magnitude of the mismatch compared to that of the frequency response of the virtual environment at the frequencies of interest.

\section{EXPERIMENTAL RESULTS}

To validate the approach presented in this paper, we consider a simple experimental set-up where interaction is performed with a 2-DoF virtual environment using a pair of Force Dimension Omega $3^{(\mathrm{TM})}$ devices [1]. Each device has 3 DoF, but only one is active while the other two are locked with mechanical clamps. Suppose two human operators interact with one device each, thus yielding the following structure of the $H-D$ interconnection in (3):

$$
\begin{aligned}
& H=\left[\begin{array}{cc}
H_{1} & 0 \\
0 & H_{2}
\end{array}\right] \in \mathscr{B} \mathscr{D}(2 ; 1,1), \\
& D=\left[\begin{array}{cc}
D_{1} & 0 \\
0 & D_{2}
\end{array}\right] \in \mathscr{B} \mathscr{D}(2 ; 1,1) .
\end{aligned}
$$

For the purpose of this experiment, the OSP levels of the devices are estimated from the damping factor of an identified first-order linear model of the Omega along one axis, yielding (in MKS units)

$$
\Delta_{D}=\left[\begin{array}{cc}
1.37 & 0 \\
0 & 1.37
\end{array}\right] .
$$

Let the virtual environment be the backward Euler discretization of the mechanical model in Fig. 4. Let the model parameters be $M=1, B_{1}=B_{2}=1, K_{1}=800, K_{2}=700$ in MKS units. The haptic system operates at a sampling time $T=1.2 \cdot 10^{-3} \mathrm{~s}$.

We simulate the presence of a transmission delay between the virtual environment simulator and the devices of 1 time step for $H_{1}-D_{1}$ and 2 time steps for $H_{2}-D_{2}$, while no environment delay is introduced, i.e, we consider

$$
\Theta\left(z^{-1}\right)=\left[\begin{array}{cc}
z^{-1} & 0 \\
0 & z^{-2}
\end{array}\right], \quad \Psi\left(z^{-1}\right)=I_{2} .
$$

A first run of the experiment is performed without introducing a virtual coupling. The corresponding time plot of device displacements is reported in Fig. 5. Self-sustained oscillatory behaviour arises during interaction, showing instability of the loop. We proceed to the synthesis of a virtual coupling using the proposed approach in two cases.

Case I. We assume

$$
\Lambda\left(z^{-1}\right)=\left[\begin{array}{cc}
z^{-1} & 0 \\
0 & z^{-2}
\end{array}\right]=\Theta\left(z^{-1}\right)
$$




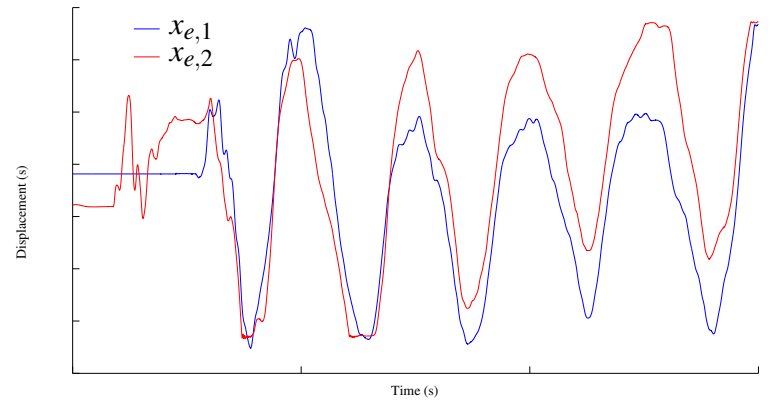

Figure 6: Device displacements during a run with virtual coupling inserted

so that $\mathscr{F}$ will be required to match a delayed version of $E$. We carry out the minimization of $\|\tilde{\mathscr{L}}\|_{\infty}$ using the procedure outlined in Section 5, where the weights $W_{1}$ and $W_{2}$ are chosen as

$$
W_{1}=I_{2}, W_{2}=w(z) I_{2},
$$

being $w(z)$ a backward Euler discretization of the low-pass filter

$$
w(s)=\frac{1}{1+\frac{s}{2 \pi f_{0}}}, f_{0}=80 \mathrm{~Hz}
$$

By introducing additional LMI constraints in the parameterization of $\mathbf{S}$ (see [7]) we also constrain $V$ to have the typical structure of a spring-damper system attached to each contact point of the virtual environment, i.e.,

$$
V(z)=\left[K+B \frac{z-1}{T z}\right]^{-1} ; K=\left[\begin{array}{cc}
k_{1} & 0 \\
0 & k_{2}
\end{array}\right], B=\left[\begin{array}{cc}
b_{1} & 0 \\
0 & b_{2}
\end{array}\right] \text {. }
$$

Minimizing $\|\tilde{\mathscr{L}}\|_{\infty}$ yields the following controller parameters:

$$
K=\left[\begin{array}{cc}
2.87 & 0 \\
0 & 2.32
\end{array}\right] \cdot 10^{3}, B=\left[\begin{array}{cc}
5.47 & 0 \\
0 & 5.55
\end{array}\right]
$$

Figure 6 depicts the evolution of the device displacements during a run of the haptic system with the virtual coupling in (26),(27) active. The plot shows the two users moving the virtual mass $M$ back and forth, but no self-sustained oscillation phenomena occur. This design achieves a transparency index (25) equal to $\tau=-4.15 \mathrm{~dB}$ in the $0-80 \mathrm{~Hz}$ frequency range.

Case II. We repeat the procedure in Case I assuming $\Lambda\left(z^{-1}\right)=I_{2}$, thus trying to steer the frequency response of $\mathscr{F}$ to match that of the undelayed virtual environment at low frequencies.

The following virtual coupling parameters are obtained:

$$
K=\left[\begin{array}{cc}
1.81 & 0 \\
0 & 0.12
\end{array}\right] \cdot 10^{3}, B=\left[\begin{array}{cc}
5.78 & 0 \\
0 & 3.81
\end{array}\right]
$$

This design yields $\tau=-3.09 \mathrm{~dB}$.

\section{Conclusion}

In this paper, the problem of virtual coupling design for multidevice, multi-operator haptic systems has been studied. A previously developed framework based on passivity and LMIs for the synthesis of stabilizing controllers has been extended to address the presence of constant time delays in the haptic loop. Moreover, a method for the design of controllers which ensure stability and transparency has been developed in this framework. The proposed approach relies on a particular loop shaping technique that involves the solution of a sequence of LMI optimization problems. Experimental validation has been conducted.

Future work will focus on taking into account more accurate models of delays and a major challenge will consist in making the approach robust with respect to variable delays. Future developments will also consist in validating the proposed approach on more complex experimental scenarios.

\section{References}

[1] Force dimension, http://www.forcedimension.com.

[2] F. Barbagli, D. Prattichizzo, and J. Salisbury. Multi-Point Physical Interaction with Real and Virtual Objects, volume 18 of STAR, Springer Tracks in Advanced Robotics. Springer Verlag, Berlin Heidelberg, 2005.

[3] F. Barbagli, D. Prattichizzo, and K. Salisbury. A multirate approach to haptic interaction with deformable objects single and multipoint contacts. International Journal of Robotics Research, 4(29):703-715, September 2005

[4] R. Bhatia and F. Kittaneh. On the singular values of a product of operators. SIAM J. Matrix Anal. Appl., 11:272-277, 1990.

[5] G. Bianchini, M. Orlandesi, and D. Prattichizzo. Analysis and design of multi-contact haptic systems: an LMI approach. In Proc. of 46th IEEE Conference on Decision and Control, pages 5761-5766, New Orleans, LA, USA, 2007.

[6] G. Bianchini, M. Orlandesi, and D. Prattichizzo. An LMI framework for analysis and design of multi-dimensional haptic systems. In Proc. of 47th IEEE Conference on Decision and Control, pages 4564-4569, Cancun, Mexico, 2008.

[7] G. Bianchini, M. Orlandesi, and D. Prattichizzo. Passivity-based analysis and design of multi-contact haptic systems via LMIs. In Advances in haptics. Intech publishing, 2010.

[8] C. Byrnes and W. Lin. Losslessness, feedback equivalence, and the global stabilization of discrete-time nonlinear systems. IEEE Transactions on Automatic Control, 39(1):83-98, 1994.

[9] J. Colgate and G. Schenkel. Passivity of a class of sampled-data systems: application to haptic interfaces. Journal of robotic systems, 14(1):37-47, 1997.

[10] J. E. Colgate, M. C. Stanley, and J. M. Brown. Issues in the haptic display of tool use. In Proc. IEEE/RSJ Int. Conf. on Robotics Systems, 1995.

[11] L. ElGhaoui, F. Oustry, and M. AitRami. A cone complementarity linearization algorithm for static output feedback and related problems. IEEE Transactions on Automatic Control, 42(8):1171-1175, 1997.

[12] M. C. J. Franken, S. Stramigioli, S. Misra, C. Secchi, and A. Macchelli. Bilateral telemanipulation with time delays: a two-layer approach combining passivity and transparency. IEEE transactions on robotics, 27(4):741-756, 2011.

[13] D. Lee and K. Huang. Passive-set-position-modulation framework for interactive robotic systems. Robotics, IEEE Transactions on, 26(2):354-369, april 2010.

[14] B. E. Miller, J. E. Colgate, and R. Freeman. Computational delay and free mode environment design for haptic display. In ASME Dyn. Syst. Cont. Div., 1999.

[15] B. E. Miller, J. E. Colgate, and R. Freeman. Guaranteed stability of haptic systems with nonlinear virtual environments. IEEE Transactions on Robotics and Automation, 16(6):712-719, 2000.

[16] B. E. Miller, J. E. Colgate, and R. Freeman. On the role of dissipation in haptic systems. IEEE Transactions on Robotics, 20(4):768-771, 2004.

[17] P. Naghshtabrizi and J. P. Hespanha. Designing transparent stabilizing haptic controllers. In IEEE American Control Conference, Minneapolis, MI, USA, 2006

[18] M. D. Oliveira, J. Camino, and R. Skelton. A convexifying algorithm for the design of structured linear controllers. In IEEE Conference on Decision and Control, pages 2781-2786, Sydney, Australia, 2000.

[19] J. Ryu, C. Preusche, B. Hannaford, and G. Hirzinger. Time domain passivity control with reference energy following. Control Systems Technology, IEEE Transactions on, 13(5):737-742, 2005.

[20] G. Sankaranarayanan and B. Hannaford. Virtual coupling schemes for position coherency in networked haptic environments. In Biomedical Robotics and Biomechatronics, 2006. BioRob 2006. The First IEEE/RAS-EMBS International Conference on, pages 853 -858, feb. 2006.

[21] S. Stramigioli, C. Secchi, A. J. van der Schaft, and C. Fantuzzi. Sampled data systems passivity and discrete port-hamiltonian systems. IEEE Transactions on Robotics, 21(4):574-586, 2005. 